CERN/ECP 95-13

27 July 1995

\title{
DESIGN STUDIES FOR A LONG BASE-LINE NEUTRINO BEAM
}

\author{
A.E. Ball \\ CERN, Geneva, Switzerland. \\ S. Katsanevas, N. Vassilopoulos*) \\ University of Athens, 104 Solonos, GR-106 80 Athens.
}

\begin{abstract}
Strong interest has recently been shown in very long base-line neutrino beams, directed at existing or planned massive detector facilities, in order to extend the search for neutrino oscillations. Among such possibilities are beams from CERN pointing towards the Gran Sasso Underground Laboratory in Italy and the NESTOR Underwater Laboratory in the Ionian Sea off the west coast of the Peloponnese.

In order to establish the basic parameters, a number of possible configurations for such beams have been studied covering a range of neutrino energy bands; estimates of the neutrino fluxes, event rates and backgrounds at typical detectors are reported. Considerations have been given to the optimum length and radius of the decay tunnels.

It is shown that with one year of operation, a neutrino oscillation search down to limits of $\sin ^{2} 2 \theta=0.01$ and $\Delta m^{2}=0.001 \mathrm{eV}^{2}$ could be made with currently proposed detectors.
\end{abstract}

To be submitted to Nuclear Instruments and Methods in Physics Research A

Presented at the Long Base-line Neutrino Meeting - Gran Sasso - December 1994.

*) Visitor at CERN - now at Oxford University 


\section{Introduction}

The probability for a two flavour neutrino oscillation is:

$$
\mathrm{P}\left(\delta m^{2}, \sin ^{2} 2 \theta\right)=\sin ^{2} 2 \theta \sin ^{2}\left(\frac{1.27 \delta m^{2} \mathrm{~L}}{\mathrm{E}_{\nu}}\right)
$$

where $\delta m^{2}=m_{1}^{2}-m_{2}^{2}$ is the difference of the mass eigenvalues in $\mathrm{eV}^{2}, \theta$ is the two flavour mixing angle, $\mathrm{L}$ is the $\nu$ propagation length in kilometres and $\mathrm{E}_{\nu}$ is the neutrino energy in $\mathrm{GeV}$.

As a rule of thumb the minimum sensitivities for the mass difference and mixing angle, for a large class of detection methods, are given approximatively by the following formulae [1]:

$$
\begin{array}{r}
\mathrm{P}_{\min }=\frac{1}{2} \sin ^{2} 2 \theta_{\min } \sim \frac{\sqrt{N}}{N} \\
\delta m_{\min }^{2} \sim \sqrt{\mathrm{P}_{\min }} \frac{<\mathrm{E}_{\nu}>}{1.27 \mathrm{~L}}
\end{array}
$$

Where $\mathrm{N}$ is the number of detected neutrino (total or Charged Current CC) events ${ }^{1 \text { ). }}$

Thus, in order to maximise sensitivity, it is necessary to have the longest possible base-line and lowest threshold energy whilst detecting sufficient neutrino events for statistical significance.

Results of the KAMIOKANDE, IMB and SOUDAN2 collaborations suggest that perhaps as much as $40 \%$ of the atmospheric $\nu_{\mu}$ events in the energy range from $0.2-1.5 \mathrm{GeV}$ have oscillated to some other type of neutrino [3],[4],[5]. On then other hand, FREJUS did not record any effect [6]. The recent 'multi-GeV' studies by KAMIOKANDE [7], have confirmed their previous result, and have even shown an azimuthal distribution of the ratio $\frac{\nu_{e}}{\nu_{\mu}}$ consistent with a $\nu_{\mu} \rightarrow \nu_{e}$ or $\nu_{\mu} \rightarrow \nu_{\tau}$ oscillation signal.

Two experiments, CHORUS [8] and NOMAD [9], looking for $\nu_{\tau}$ appearance in the present CERN wide-band neutrino beam are currently taking data; some results are expected in 1995/6.

Clearly one would like to continue with controlled beam experiments because they have the following advantages over atmospheric data:

- Initial flavour composition well known (typically $\nu_{e}$ is $1 \%$ of $\nu_{\mu}$ ),

- Control of the beam polarity. One can switch between $\nu$ and $\bar{\nu}$ beams to study matter enhanced oscillation (MSW) effects [10]).

- Control of the energy. One can typically obtain beam energy dispersions of $\sigma_{E} \sim 5 \mathrm{GeV}$

- One can assume the direction cosines and time of arrival of the neutrino, improving efficiency and reducing backgrounds substantially

- Higher statistics, giving sensitivity to lower mixing angles

- Control of the beam energy, providing a method to differentiate between oscillations from $\nu_{\mu}$ to $\nu_{e}$ or to $\nu_{\tau}$.

\section{General design considerations}

Neutrino beams from proton accelerators are essentially $\nu_{\mu}$ from the decay of pions and kaons, produced when an extracted beam strikes an external target. These 'parent' particles are focused towards the detector and left to decay in an evacuated tunnel to produce muons and $\mu$ neutrinos. The muons and the remaining hadrons are stopped by shielding at the end of the decay tunnel leaving only the neutrinos to continue to the detector. A smaller fraction of $\nu_{e}$ are also produced from kaon decays and from secondary decays of muons, giving an unwanted background in $\nu_{\mu} \rightarrow \nu_{e}$ searches.

\footnotetext{
1) For a detailed discussion of the exact numerical factors that enter in the above formulae, see e.g [2]
} 
The ratio of pions to kaons produced from protons interacting in the target is roughly 10:1. The mean free path for pions is about $55 \mathrm{~m} / \mathrm{GeV}$ and for kaons around $7.5 \mathrm{~m} / \mathrm{GeV}$. The average neutrino energy produced in pion decay is some $43 \%$ of the pion energy whereas for kaons the neutrino has, on average, some $95 \%$ of the parent kaon energy. Thus for a decay path of some 600 metres only $40 \%$ of the $23 \mathrm{GeV}$ pions will have decayed to give neutrinos of about $10 \mathrm{GeV}$ whereas essentially all kaons of $10 \mathrm{GeV}$ will have decayed. This leads essentially to two superimposed $\nu_{\mu}$ energy spectra, dominated by the pion generated contribution at lower energies with a smaller kaon contribution at higher energy.

In order to obtain acceptable neutrino event rates at a detector many hundreds of kilometres from the source, it is essential to have an efficient target in which most of the incident protons interact but which, at the same time, gives minimal absorption and scattering to the hadrons produced. The focusing system must have a large acceptance for the parent pions and kaons in the required energy range and produce a beam as nearly parallel as possible; even so, the flux at the detector is largely dominated by the decay kinematics of neutrino production.

This study concentrates on co-axial magnetic lenses (so-called horns and reflectors) since they give substantially higher angular and momentum acceptance at lower energies than can usually be achieved with quadrupole lenses; they also give particle sign selection which in-line quadrupoles do not. However some simulations of quadrupole triplet focusing are made and a possible application is considered.

Specific narrow energy band systems have not been studied at present since the reduction in flux due to tight momentum selection seems unacceptable for currently considered detectors. Other focusing systems such as plasma or lithium lenses can, in theory, have some advantages over 'conventional' horns but usually have limited angular acceptance and may not have the same level of long-term reliability in operation.

A prime consideration in the design of a neutrino beam is the very high level of induced radioactivity around the target station and focusing system; to ensure reliability and minimise personnel exposure, it is essential to avoid all organic compounds and materials giving rise to long half-life decay products or contamination.

Interest in a long baseline neutrino beam from CERN has been stimulated by three factors:

- as part of the LHC project at CERN, new transfer lines are required to bring fast extracted protons from the SPS to the LHC; the detailed layout is still under study but it has been shown [11] that it would be possible to derive a neutrino beam from the TI 87 line linking SPS-LSS4 to the LHC/LEP ring near point 8;

- during the period of LHC installation it may be possible to run the SPS machine in an essentially dedicated mode with substantially shorter duty cycles; later, during LHC operation, an SPS beam would be available outside the LHC filling periods;

- two substantial neutrino detector facilities exist or are under development which lie in the general direction of such a beam:

- the Gran Sasso underground laboratory (in particular the ICARUS detector[12]) at a distance of $731 \mathrm{~km}$.

- the deep underwater neutrino laboratory NESTOR [13] in the Mediterranean at a distance of $1676 \mathrm{~km}$.

Table 1 [14] gives the absolute co-ordinates of CERN and these detector sites showing that the directions from CERN are within 1.68 degrees in azimuth and 5.24 degrees in declination. The possibility of providing neutrino beams to both of these facilities using common or shared beam equipment is clearly attractive; the additional cost of providing a double facility is principally in the civil engineering cost of an enlarged section of the transfer line and the two decay tunnels.

It is also worth noting that possibilities of long base-line beams are under active study in the USA and Japan: HEPAP reviews were conducted during June 1995 both at Fermilab 


\begin{tabular}{|l|c|c|c|c|c|}
\hline \hline Place & $\lambda$ & $\phi$ & $\mathrm{A}_{z}$ & $\alpha$ & Distance \\
\hline CERN & 6.0732 & 46.2442 & - & - & - \\
\hline Gran Sasso & 13.5744 & 42.4525 & 122.502 & 3.283 & $731 \mathrm{~km}$ \\
\hline Nestor & 21.3500 & 36.3500 & 124.1775 & 8.526 & $1676 \mathrm{~km}$ \\
\hline \hline
\end{tabular}

Table 1: Absolute coordinates $(\lambda, \phi)$ and azimuth and declination angles $\left(A_{z}, \alpha\right)$ in degrees, of Gran Sasso and Nestor w.r.t CERN

and Brookhaven. One proposal is for a beam from the Fermilab $120 \mathrm{GeV}$ Main Injector to the SOUDAN site at $732 \mathrm{~km}$ distance (MINOS proposal) [15]. There are also two "lower energy" beams proposed: the first from the $24 \mathrm{GeV}$ Brookhaven AGS to sites up to $30 \mathrm{~km}$ on Long-Island [16] and the second in Japan from KEK (12 GeV) to Superkamiokande at $250 \mathrm{~km}$ [17].

\section{Beam simulation}

The version 3.21 of GEANT [18] has been used throughout this study to simulate the beam line; realistic values for material thicknesses, magnetic field strengths, etc. have been used; the only exception to this is where the notion of so called "perfect focusing" has been noted.

The graphics capabilities of GEANT ++ proved extremely useful and the debugging and optimization tools were used extensively in the design. Using ray tracing techniques, an example of which is seen in figure 2, the behaviour of the focusing elements could be studied before launching the very long runs for flux and event rate calculations. Figure 3 shows clearly the focusing and defocusing effects of the combined $20 \mathrm{GeV} / c$ horn and $40 \mathrm{GeV} / c$ reflector on positive and negative parent particles.

For the remainder of this study two typical detectors are considered:

- a detector having a cross-sectional area of 20×20 $\mathrm{m}^{2}$ at the Gran Sasso site situated at 731 $\mathrm{km}$ from CERN; note that the distance from FNAL to the MINOS/SOUDAN2 detector is $732 \mathrm{Km}$.

- a NESTOR detector having an area of $40 \times 240 \mathrm{~m}^{2}$ situated at $1676 \mathrm{~km}$ from CERN; this corresponds to a single tower as presently foreseen for astrophysics research; a larger array would be considered for a specific beam experiment.

The proton beam is assumed to have a normal intensity distribution with $\sigma=0.5 \mathrm{~mm}$ at a beryllium target of $3 \mathrm{~mm}$ diameter; different incident proton beam energies and beam configurations are examined. To reduce statistical fluctuations, half a million proton interactions in the target are used.

The products of the hadronic interactions are tracked through the optical system, focused and allowed to decay. Secondary interactions, absorption and multiple scattering in all materials are included. Each decay occuring within the beam cavern or decay tunnel generates 1000 neutrinos which are projected to the detector plane; fluxes and event rates in the detectors are weighted accordingly and there is no evidence that this introduces significant systematic errors ${ }^{2}$.

The tables and plots for neutrino flux are renormalised to $10^{13}$ protons on target, and event rate per kiloton to $10^{19}$ protons on target, which roughly corresponds to a good year of running at present duty cycles.

\footnotetext{
2) Control rums with only 100 neutrinos per hadron decay give the same results within statistical variations.
} 


\section{Comparison of production models}

A major systematic uncertainty in estimating neutrino fluxes is the model of hadronic interactions used to obtain the production spectra. This is particularly true at energies which are low compared to the incident proton energy and where the production is dominated by cascade processes. Three production models have been compared, GHEISHA [19], FLUKA [20] and a thermodynamic model using fitting parameters from Atherton et al. ( $\left.\mathrm{E}_{p}>200 \mathrm{GeV}\right)[21]$ and Barton et al. $\left(\mathrm{E}_{p}<200 \mathrm{GeV}\right)[22]$

For this, "perfect focusing" and a 20x20 $\mathrm{m}^{2}$ detector at Gran Sasso with 100\% detection efficiency are assumed (table 2, and figure 1). In perfect focusing, the hadronic secondaries are forced to be parallel to the beam axis at the entrance of a 3 metre radius decay tunnel which starts at 2 metres behind the target centre, thus giving a fixed acceptance from the target of about 1.5 radians. The target used for this study consisted of 6 beryllium rods of $1.5 \mathrm{~mm}$ radius and $10 \mathrm{~cm}$ length with $5 \mathrm{~cm}$ gaps between $\operatorname{rods}^{3)}$.

\begin{tabular}{|l|c|c|c|}
\hline \hline $\mathrm{E}_{p}$ & Thermo & FLUKA & GHEISHA \\
\hline $\mathrm{GeV}$ & \multicolumn{3}{|c|}{$\nu$ fluxes $/ 10^{13} \mathrm{p} / \mathrm{m}^{2}$} \\
\hline 80 & $1.910^{4}$ & $2.710^{4}$ & $2.410^{4}$ \\
\hline 160 & $5.110^{4}$ & $7.510^{4}$ & $7.910^{4}$ \\
\hline 400 & $1.610^{5}$ & $2.410^{5}$ & $2.810^{5}$ \\
\hline $\mathrm{GeV}$ & \multicolumn{3}{|c|}{ Events $/ 10^{19} \mathrm{p} / \mathrm{kton}$} \\
\hline 80 & 81 & 120 & 110 \\
\hline 160 & 387 & 568 & 709 \\
\hline 400 & 2270 & 3215 & 4260 \\
\hline $\mathrm{GeV}$ & \multicolumn{3}{|c|}{$<E_{\nu}^{-2}>^{-\frac{1}{2}}$ in $\mathrm{GeV}$} \\
\hline 80 & 7.5 & 6.7 & 6.2 \\
\hline 160 & 12.8 & 10.9 & 14. \\
\hline 400 & 24.9 & 18.1 & 19.7 \\
\hline
\end{tabular}

Table 2: Neutrino fluxes, event rates (for $100 \%$ detection efficiency) and neutrino mean energy with perfect focusing and fixed acceptance for three hadronic production models; a detector of cross sectional area $20 \times 20 \mathrm{~m}^{2}$ at Gran Sasso is assumed

The thermodynamic model, widely used for previous neutrino beam calculations at CERN, gives consistently $50 \%$ less flux and number of events than FLUKA; In fact the two models are consistent down to $15 \%$ of the incident beam energy, below which the thermodynamic model predicts much lower fluxes. This is not unexpected since no attempt was made to fit parameters to experimental data below $25 \%$. Production spectra from GHEISHA are closer to FLUKA but show oscillations; they give slightly more flux at the higher energies.

Recent versions of FLUKA are thought to reproduce the low energy part of the production spectrum correctly and comparisons made with radiation dose and fluence measurements [23], which are particularly sensitive to cascade mechanisms, show good agreement.

However, without experimental particle production data at energies well below that of the incident beam, it is not possible to conclude that any one model simulates correctly the production or the $\mathrm{K} / \pi$ ratios. In the energy range of interest, there is some evidence that FLUKA is the better model and it is the principal model used in LHC hadronic simulations; it has therefore been used for the remainder of this study in order to compare different optical systems; however the uncertainty in quoted fluxes and event rates must be considered to be of the order of $20-25 \%$.

3) Different geometries are used in subsequent calculations; the numbers in table 2 should be used only for the comparison of production models. 


\section{Beam line elements}

Simulations of the beam line take as origin $\left(\mathrm{Z}_{0}\right)$ the approximate centre of production in an extended target; this is used as the focal point for calculating the initial parameters of the focusing system. The elements used are:

\section{- Segmented cylindrical beryllium target.}

Targets of $80-120 \mathrm{~cm}$ overall length are made up from rods of $10 \mathrm{~cm}$ length and $1.5 \mathrm{~mm}$ diameter with gaps of $5 \mathrm{~cm}$ between rods; such targets have been used for many years in neutrino beams at CERN. Segmenting the target avoids the development of thermal shock waves which can cause fracture and enhances target cooling; lowering the effective density also improves the low energy secondary production by reducing absorption and scattering in the target material. No attempt has been made at present to optimise the target layout for given focusing configurations.

\section{- Focusing system - magnetic horns and reflectors.}

The horn and reflector are co-axial lenses with a radial field between the inner and outer conductors; a current of $110 \mathrm{kA}$ during the beam pulse has been assumed. The inner conductor of the horn has a roughly ellipsoid form, made up of 8 conical pieces; 3 horn shapes, designed to focus tracks of 15,20 and $40 \mathrm{GeV} / c$ momentum, have been used (H15, $\mathrm{H} 20, \mathrm{H} 40$ ). The horns start at $60 \mathrm{~cm}$ from $\left(\mathrm{Z}_{0}\right)$ and have an overall length of $290 \mathrm{~cm}$ (see figure 2).

The reflector is similar but has a central aperture designed to allow the well focused parent particles from the horn to pass undisturbed while providing additional focusing to under and over-focused particles; the inner conductor consists of 4 conical sub-elements. Four reflectors shapes have been used which, when combined with the appropriate horn, focus tracks of $20,25,40$, and $70 \mathrm{GeV} / c$ momentum (R20, R25, R40, R70). The reflectors start at $1050 \mathrm{~cm}$ from $\left(\mathrm{Z}_{0}\right)$ and have an overall length of $600 \mathrm{~cm}$.

As the required neutrino energy band is low compared to the incident beam energies, a particular aim in this study has been to design compact focusing systems, close to the target, which give high angular acceptance of the parent muons. The outer conductor dimensions can be made sufficiently large so as to give no significant additional aperture limit.

\section{- Focusing system - quadrupole triplets.}

An alternative focusing system consisting of magnetic quadrupole lenses in a triplet configuration has also been considered. Arranged with alternate focusing-defocusing-focusing polarities and with the central element having approximately twice the strength (or length) of the outer lenses, a triplet can behave as a thin lens. For realistic field strengths and dimensions, such systems have a non-isotropic and in general lower angular acceptance than horn/reflector systems but, since they have no materiel within the effective aperture, there is no absorption and scattering of the focused particles. Quadrupole triplets give no intrinsic sign selection between positive or negative parent particles but differences in acceptance can affect the relative fluxes if there are other dispersive elements later in the beam line.

Triplet configurations have been simulated with nominal focusing for 20,30 and $40 \mathrm{GeV} / c$ tracks. The Q20 system has magnets of 180 and $360 \mathrm{~cm}$ magnetic length and $30 \mathrm{~cm}$ radius aperture; the first magnet starts at $49 \mathrm{~cm}$ from $\left(\mathrm{Z}_{0}\right)$ and the inter-magnet spacing is 49 $\mathrm{cm}$. The Q40 system has only $20 \mathrm{~cm}$ aperture and is slightly longer. The Q30 system uses the same magnets as Q20 but increased inter-magnet spacing. The maximum field on the pole tip is 1.5 Tesla in all systems.

- Decay tunnel The decay path is an evacuated cylinder, with maximum dimensions of $1000 \mathrm{~m}$ length and $2 \mathrm{~m}$ radius. The begining of the decay tunnel is at 25 metres from $\left(\mathrm{Z}_{0}\right)$ and has a $2 \mathrm{~mm}$ thick titanium window. Decays can of course occur anywhere after 
the target and are included in the calculations. The co-ordinates of the decay points of particles generating neutrinos are stored so that the effect of changing the length and radius of the decay tunnel can be examined.There is, however no tracking of particles other than neutrinos outside or beyond the limits of the decay tunnel.

\section{Fluxes, event rates and backgrounds}

\section{Horn and Reflector focusing}

Tables 3 and 4 give the $\nu_{\mu}$ flux and event rates, neutrino mean energy and fractional background of $\nu_{e}$ for detectors situated at the Gran Sasso and NESTOR sites for a number of incident proton energies and focusing systems ${ }^{4)}$. Proton beam energies of 80 and $160 \mathrm{GeV} / c$ have been considered in a number of senarios where one might benefit from a higher repetition rate from the accelerator by running a lower peak energy; the energy of $120 \mathrm{GeV} / c$ is included for comparison with studies of the Fermilab Main Injector beam to SOUDAN2/MINOS.

\begin{tabular}{|c|c|c|c|c|c|c|c|c|c|}
\hline $\mathrm{E}_{p}$ & $\mathrm{PF}$ & H15 & H15R25 & $\mathrm{H} 20$ & H20R20 & H20R40 & H20R40a & H40R70 & WANF \\
\hline $\mathrm{GeV}$ & \multicolumn{9}{|c|}{$\nu$ fluxes in $10^{4} \nu / 10^{13} \mathrm{p} / \mathrm{m}^{2}$} \\
\hline 80 & $\begin{array}{ll}3.7 \\
\end{array}$ & 0.6 & 1.1 & 0.6 & 0.96 & 0.8 & & & \\
\hline 120 & 6.0 & 0.9 & 1.6 & 1.1 & 1.4 & 1.6 & & & \\
\hline 160 & 9.0 & 1.3 & 2.1 & 1.5 & 2.1 & 2.6 & & & \\
\hline 450 & 31.0 & 3.9 & 7. & 4.7 & 6.5 & 7.6 & $(0.97)$ & 8.4 & 2.3 \\
\hline $\mathrm{GeV}$ & \multicolumn{9}{|c|}{$\nu_{\mu}$ Events $/ 10^{19} \mathrm{p} / \mathrm{kt}$} \\
\hline 80 & 151 & 19 & 38 & 24 & 38 & 32 & & & \\
\hline 120 & 341 & 32 & 65 & 44 & 55 & 77 & & & \\
\hline 160 & 578 & 55 & 90 & 63 & 86 & 133 & & & \\
\hline 450 & 3516 & 236 & 390 & 292 & 338 & 473 & 130 & 722 & 357 \\
\hline $\mathrm{GeV}$ & \multicolumn{9}{|c|}{$\left\langle E_{\nu}^{-2}\right\rangle^{-\frac{1}{2}}$ in $\mathrm{GeV}$} \\
\hline 80 & 6.8 & 5.8 & 7.0 & 6.7 & 6.5 & 6.5 & & & \\
\hline 120 & 8.6 & 5.8 & 7.0 & 7.2 & 7.1 & 8.3 & & & \\
\hline 160 & 9.3 & 7.3 & 8.0 & 7.2 & 7.1 & 8.9 & & & \\
\hline 450 & 14.6 & 8.5 & 9.4 & 9.5 & 8.8 & 10.5 & $(16.2)$ & 14.2 & 21.4 \\
\hline $\mathrm{GeV}$ & \multicolumn{9}{|c|}{$\nu_{e} / \nu_{\mu}(\%)$} \\
\hline 80 & 0.66 & 1.00 & 0.74 & 0.68 & 0.78 & 0.60 & & & \\
\hline 120 & 0.57 & 1.17 & 0.78 & 1.00 & 0.92 & 0.88 & & & \\
\hline 160 & 0.50 & 1.18 & 1.20 & 1.00 & 0.93 & 1.00 & & & \\
\hline 450 & 0.50 & 1.96 & 1.57 & 2.00 & 1.58 & 1.40 & $(2.66)$ & 1.11 & \\
\hline $\mathrm{GeV}$ & \multicolumn{9}{|c|}{$\bar{\nu}_{\mu}$ Events $/ 10^{19} \mathrm{p} / \mathrm{kt}$} \\
\hline 80 & 50 & 0.6 & 0.5 & 0.6 & 0.5 & 0.6 & & & \\
\hline 120 & 115 & 2 & 1.5 & 2 & 1.75 & 1.9 & & & \\
\hline 160 & 210 & 5 & 4 & 4 & 4 & 4 & & & \\
\hline 450 & 1500 & 45 & 52 & 50 & 51 & 47 & 213 & 33 & 0 \\
\hline
\end{tabular}

Table 3: Rates at Gran Sasso for different focusing geometries and beam energies. The numbers of events in parentheses concern the $\bar{\nu}_{\mu}$ rates.

The energy of the incident protons determines the overall envelope of the neutrino spectum and has the greatest influence on the flux of neutrinos produced at all energies. Operating at the highest available energy is clearly the most efficient and cannot be compensated by even tripling the repetition rate at lower beam energies.

The PF (perfect focusing) data is included to show the idealistic flux one would obtain if all mesons entering the horn aperture could be focused parallel to the axis without absorption or scattering; note that in this case the negative mesons are also focused whereas in the real focusing systems the "wrong sign" mesons are defocused.

\footnotetext{
4) assuming $100 \%$ detection efficiency
} 


\begin{tabular}{|c|c|c|c|c|c|c|c|c|c|}
\hline$\overline{\mathrm{E}_{p}}$ & $\overline{\mathrm{PF}}$ & $\mathrm{H} 15$ & H15R25 & $\mathrm{H} 20$ & $\mathrm{H} 20 \mathrm{R} 20$ & H20R40 & H20R40a & H40R70 & WANF \\
\hline $\mathrm{GeV}$ & \multicolumn{9}{|c|}{$\nu$ fluxes in $10^{3} \nu / 10^{13} \mathrm{p} / \mathrm{m}^{2}$} \\
\hline 80 & 6.7 & 1.1 & 1.7 & 1.1 & 1.7 & 1.7 & & & \\
\hline 120 & 11.0 & 1.8 & 2.9 & 2.1 & 2.8 & 3.1 & & & \\
\hline 160 & 17.0 & 2.5 & 4.2 & 2.8 & 4. & 4.6 & & & \\
\hline 450 & 58.0 & 7.5 & 13.9 & 9.2 & 12. & 15. & $(1.8)$ & 16. & 4.6 \\
\hline $\mathrm{GeV}$ & \multicolumn{9}{|c|}{$\nu_{\mu}$ Events $/ 10^{19} \mathrm{p} / \mathrm{kt}$} \\
\hline 80 & 27 & 4 & 7 & 4 & 7 & 8 & & & \\
\hline 120 & 61 & 7 & 12 & 8 & 11 & 15 & & & \\
\hline 160 & 116 & 11 & 19 & 12 & 16 & 23 & & & \\
\hline 450 & 657 & 44 & 72 & 55 & 64 & 93 & 24 & 134 & 71 \\
\hline $\mathrm{GeV}$ & \multicolumn{9}{|c|}{$\left\langle E_{\nu}^{-2}\right\rangle^{-\frac{1}{2}}$ in $\mathrm{GeV}$} \\
\hline 80 & 6.3 & 5.7 & 6.9 & 6.1 & 6.3 & 7.5 & & & \\
\hline 120 & 7.7 & 5.9 & 7.6 & 7.0 & 7.2 & 8.1 & & & \\
\hline 160 & 9.7 & 9.3 & 8.0 & 7.6 & 7.3 & 9.3 & & & \\
\hline 450 & 14.6 & 8.7 & 9.4 & 9.5 & 9.0 & 10.7 & $(17.5)$ & 14.6 & 20.6 \\
\hline $\mathrm{GeV}$ & \multicolumn{9}{|c|}{$\nu_{e} / \nu_{\mu}(\%)$} \\
\hline 80 & 0.66 & 1.00 & 0.14 & 1.00 & 0.83 & 0.74 & & & \\
\hline 120 & 0.61 & 1.15 & 0.77 & 1.00 & 1.00 & 1.00 & & & \\
\hline 160 & 0.50 & 1.18 & 1.25 & 1.00 & 1.00 & 1.00 & & & \\
\hline 450 & 0.50 & 2.20 & 1.57 & 2.50 & 1.71 & 1.50 & $(2.66)$ & 1.20 & \\
\hline $\mathrm{GeV}$ & \multicolumn{9}{|c|}{$\bar{\nu}_{\mu}$ Events $/ 10^{19} \mathrm{p} / \mathrm{kt}$} \\
\hline 80 & 9 & 4 & 0 & 0 & 0 & 0 & & & \\
\hline 120 & 22 & 0.4 & 0.2 & 0.35 & 0.35 & 0.35 & & & \\
\hline 160 & 40 & 0.8 & 0.7 & 0.9 & 0.8 & 0.75 & & & \\
\hline 450 & 300 & 5 & 10 & 8 & 9 & 8 & 38 & 6 & 0 \\
\hline
\end{tabular}

Table 4: Rates at NESTOR for different focusing geometries and beam energies. The numbers of events in parentheses concern the $\bar{\nu}_{\mu}$ rates.

The function of the focusing system is to select a particular neutrino energy band from the overall production spectrum and deliver this to the detector as efficiently as possible. The tables show the rates for a number of systems with horn only or horn and reflector combined; while the variants shown are clearly not exaustive they serve to show the main possibilities of co-axial lens systems and how the reflector can be used to increase the flux by broadening the momentum acceptance at higher or, in the case of the H20R20 system, lower energies.

Of the different focusing systems considered it would seem that the $20 \mathrm{GeV} / c$ horn and $40 \mathrm{GeV} / c$ reflector system (H20R40) gives the best compromise between the flux/event rate obtained and the mean energy of the neutrino spectrum in order to obtain good sensitivity to oscillations with the expected detector efficiencies.

Compared with perfect focusing it can be seen that real focusing systems can deliver 22 $29 \%$ of the 'available' neutrino flux to the detector; this compares favorably with other neutrino beams and indicates that the focusing system is well matched to the production; however, when comparing event rates, the efficiency of the focusing system at lower proton energies is around $20-25 \%$ whilst it is only $13-14 \%$ at $450 \mathrm{GeV}$.

Figure 4 shows the energy spectrum for neutrino events at the NESTOR detector for incident proton energies of 120 and $450 \mathrm{GeV}$. At $120 \mathrm{GeV}$ the focusing system gathers a large fraction of the available "perfect" spectum which falls off above $30 \mathrm{GeV}$; however at $450 \mathrm{GeV}$ the fraction collected is much smaller because events in the high energy "tail" have a higher detection cross-section.

Figure 5 shows that it possible to design focusing systems which select different energy "bites" from the available production spectrum. The rates for the H40R70 system are given in the tables for $450 \mathrm{GeV}$ incident protons only. 
The H15R25 system picks up the lowest energies within the production envelope whereas the H40R70 system gives a $50 \%$ higher event rate than the H20R40 system but the neutrino mean energy is increased by $35 \%$. Thus by changing the focusing configuration, the neutrino beam produced can be tuned in energy through the 'available' spectrum.

The tables also give the yields obtained when the polarity of the H20R40 system is reversed (H20R40a) so as to focus the anti-neutrino parents; the defocused neutrino component is still significant but could be greatly reduced by an axial absorber. For comparison, these tables also give the calculated rates obtained by extrapolating the West Area Neutrino Facility (WANF) beam presently running at CERN for the CHORUS and NOMAD experiments which is designed focus a much higher energy band; the flux is only $30 \%$ of the H20R40 system but one obtains $75 \%$ of the event rate since the lower flux is largely compensated by the doubling of the mean energy.

\section{Quadrupole focusing}

Table 5 and fig 5 show the results obtained using quadrupole triplet structures with an incident proton beam of $450 \mathrm{GeV}$.

\begin{tabular}{|l|c|c|c|c|}
\hline \hline geometry & $\nu$ fluxes & Events & $\left\langle E_{\nu}^{-2}\right\rangle^{-\frac{1}{2}}$ & $\nu_{e} / \nu_{\mu}(\%)$ \\
\hline Q20 GS & 34 & $278(96)$ & 13.5 & 2.18 \\
\hline Q30 GS & 37 & $320(120)$ & 14.3 & 2.00 \\
\hline Q40 GS & 42 & $386(151)$ & 17.7 & 1.25 \\
\hline Q20 NESTOR & 6.6 & $55(21)$ & 13.9 & 2.01 \\
\hline Q30 NESTOR & 7.2 & $63(23)$ & 14.5 & 1.90 \\
\hline Q40 NESTOR & 8.3 & $83(27)$ & 19.3 & 1.33 \\
\hline
\end{tabular}

Table 5: Gran Sasso and Nestor rates with 3 different quadrupole geometries, for a $450 \mathrm{GeV} p$ beam. The numbers of events in parentheses give the $\bar{\nu}_{\mu}$ rates. The $\nu_{e}$ to $\nu_{\mu}$ ratio is for the sum of both neutrinos and antineutrinos. Fluxes are in units of $10^{4} \nu / 10^{13} \mathrm{p} / \mathrm{m}^{2}$ and events are given per kiloton for $10^{19}$ protons on target.

The combined $\nu_{\mu}$ and $\bar{\nu}_{\mu}$ fluxes obtained are roughly equivalent to the yield from a single horn system without reflector; the event rate is comparable to horn/reflector systems because of the higher mean energies and $\nu_{e}$ backgrounds are in general higher. There is obviously no control of the beam polarity. There seems to be no good reason to prefer quadrupole focusing over co-axial lens systems, particularly when technical problems such as coil insulation in the high radiation area close to the target are taken into consideration, except that the quadrupole focused systems are sufficiently momentum selective as to provide a good approach to narrowband beams.

A possible application of quadrupole triplet systems has been examined which uses the absence of sign selection. The beam configuration consists of a triplet structure(Q20), as above, followed by a decay tunnel of some 100 metres, set on an axis which bisects the angles to Gran Sasso and NESTOR. A large dipole magnet then to sweeps the $20 \mathrm{GeV} \pi+$ and $\pi$ - beams into further decay tunnels pointing towards the two detector sites. Most of the kaons will have decayed in the first 100 meters of decay tunnel, so that a principal source of $\nu_{e}$ background in the detectors is eliminated, and the system provides two beams of $\nu_{\mu}$ and $\bar{\nu}_{\mu}$, with less than $0.3 \% \nu_{e}$ background, simultaneously to the two detector sites.

However, as might be expected, the dispersion caused by the dipole magnet is such that only $5 \%$ of the flux reaches the detectors compared to a beam pointing directly at them. Achromatic bending systems can be made or additional focusing in the secondary decay tunnels could be considered, but it is difficult to conceive of such devices having the large apertures that would be required. In addition, such a tunnel would not be compatible with conventional beam layouts. 


\section{Radial beam dimensions}

The radial distribution of events at the two detector sites with a horn/reflector focusing system (H20R40) is shown in figure 6 for incident proton beams of 80,120 and $450 \mathrm{GeV}$. The distributions are flat over distances which are large compared to the detector dimensions with PWHM radii $\sim 2 \mathrm{~km}$ at Gran Sasso and $\sim 5 \mathrm{~km}$ at NESTOR. The NESTOR rates shown are multiplied by 5.244, the square of the ratio of Gran Sasso and NESTOR distances. The exact matching at small radii of events rates (fig 6 ) and energy spectra (fig 7 ) suggests that at these large distances and within $1 \mathrm{mrad}$, the beam system behaves exactly as a point source. Figure 8 shows that over radial dimensions $\sim 1 \mathrm{~km}$, there is no evidence of fluctuations in beam energy or composition; this suggest that integrating fluxes and event rates over $1 \mathrm{~km}$ at Gran Sasso or $2 \mathrm{~km}$ at NESTOR in future calculations will not induce significant systematic errors.

These considerations also indicate that the beam direction should be maintained within 2 milliradians; alignment of the beam elements and the proton beam to within one milliradian is not considered a problem.

\section{Dimensions of the decay tunnel}

The length and radius of the decay tunnel are parameters which have important implications for the civil engineering and the cost of a neutrino beam facility as well as on the beam quality.

Figure 9 shows the integrated fraction of $\nu_{\mu}$ neutrinos that pass through the fiducial area of NESTOR, as a function of the radial distance $R$ from the beam axis and the distance $\mathrm{z}$ along this axis of the decay point.

One sees that a decay tunnel of $\sim 1.5 \mathrm{~m}$ radius contains $95 \%$ of decays that generate events within the detector acceptance. However, reducing the decay tunnel length from 1000 to $\sim 800$ metres would reduce the number of events detected by $10 \%$ giving $420(83) \mathrm{CC} \nu_{\mu}$ events $/ 10^{19} / \mathrm{kt}$ for GS(NESTOR).

\section{$\nu_{e}$ background}

However, another important element that enters into considerations of the decay tunnel length is the $\nu_{e}$ contamination in the beam. Tables 3 and 4 show that the ratio of $\nu_{e}$ to $\nu_{\mu}$ is $\sim 1 \%$, rising to $1.5 \%$ at $450 \mathrm{GeV} / c$; it is the main systematic error in the analysis, setting sensitivity limits on the mixing angle, for $\nu_{\mu}$ to $\nu_{e}$ oscillations.

For $450 \mathrm{GeV}$ incident beam energy and an H20R40 focused $\nu_{\mu}$ beam, figure 10 shows the energy distribution of neutrinos that fall within the NESTOR acceptance and the z co-ordinate along the beam axis of the decay point of the parent particle. In order to examine the various sources of the background, the $\nu_{e}$ from $\mu, K_{+}$and $K_{0}$ decays are shown separately.

The $\nu_{e}$ events coming from kaon decay are uniformly spread in energy and are produced early in the decay tunnel, as expected from the short kaon lifetime. The $\nu_{e}$ events coming from decays of $\mu$, which make up a non-negligable fraction of the $\nu_{e}$ background, are concentrated in the low energy region and are mainly produced at large $\mathrm{Z}$ distances in the decay tunnel since they are products of secondary decays.

There is some indication that the fraction of $\nu_{e}$ in $\nu_{\mu}$, which initially falls with increasing $\mathrm{Z}$, starts to rise again after some 800 metres due to the $\mu$ contribution. It would be possible to reduce the effective $\nu_{e}$ background from the kaon decays by applying an upper energy cut of $\sim$ $25 \mathrm{GeV}$ in the analysis without a major effect on the $\nu_{\mu}$ rate. It might, however, be reasonable to limit the length of the decay path to $800 \mathrm{~m}$ in order to reduce the low energy background from $\mu$ decay. These considerations apply equally to a detector at Gran Sasso since the solid angles from the source are equivalent. 


\section{Conclusions}

- It has been shown that a realistic, compact beam can be designed using horn and reflector focusing which, with an 800 metre long decay tunnel of 1.5 metre radius, will produce a low energy neutrino beam providing typically $\sim 7-10$ thousand events per year at the detector sites with $10^{19}$ protons on target at $450 \mathrm{GeV}$.

- The energy dispersion of such a beam is $\sim \pm 5 \mathrm{GeV}$ and the mean neutrino energy can be tuned to between 8 and $20 \mathrm{GeV}$ by selecting the focusing elements (see fig 5). The higher mean energies and improved statistics are necessary for $\nu_{\tau}$ oscillation studies, provided one accepts the relative loss in sensitivity in $\Delta m^{2}$. Lower energy dispersion could be achieved with quadrupole focusing.

- The $\nu_{e}$ contamination is a limiting factor for the mixing angle sensitivity and is at the level of $1 \%$ of the $\nu_{\mu}$ rate.

- The beam dispersion is such that one can confidently target the detector with a beam alignment accuracy of 1 milliradian.

- The incident proton beam energy should be the highest available; lower energies considerably reduce the neutrino flux which can only partially be compensated by a higher repetition rate from the accelerator and would also limit the possibilities for $\nu_{\tau}$ oscillation studies. ${ }^{5)}$

- No detailed studies of target geometry and efficiency are reported since this is regarded as premature; a programme of theoretical studies and beam tests on target materials, configurations and cooling, aimed at increasing the intensity to possibly $3.10^{13}$ protons per pulse, is giving promising results.

- Making a detailed study and comparison of detector efficiencies is beyond the scope of this paper. However some indication of the expected sensitivity can be given.

- With $10^{19}$ protons of $450 \mathrm{GeV} / c$ one can realistically hope to obtain $\sim 500$ events/kiloton at Gran Sasso; a 15 kiloton detector with $100 \%$ efficiency would collect $\sim 7500$ events per year.

- A single NESTOR tower is more than $200 \mathrm{kton}$; with a dense phototube configuration one expects a $50 \%$ detection efficiency with a $2 \mathrm{GeV}$ threshold. With $\sim 100$ events/kiloton one would could collect considerably more than 10000 events per year.

With these statistics one would obtain a $1 \%$ statistical sensitivity for the oscillation mixing angle according to equation (2). The $\Delta m^{2}$ sensitivity, essentially determined by the lever arm and the minimum detectable neutrino energy, is of the order of $10^{-3} \mathrm{eV}^{2}$ for Gran Sasso and NESTOR. These limits amply cover the reported KAMIOKANDE signal. With identical beams, detectors at Gran Sasso and NESTOR sites could be completely complementary in an oscillation search.

\footnotetext{
5) Recent work suggests that operating the SPS at $400 \mathrm{GeV} / \mathrm{c}$ will give the most efficient production duty cycle
} 


\section{References}

[1] See e.g S. J. PARKE, FERMILAB Conf-91/251-T September 1991, M. Goodman, ANLHEP CP-92-17, Summary talk of the Workshop on Long Baseline Neutrino Oscillations, Fermilab, November 17-20, 1991, R.Bernstein, FERMILAB-Conf-92/63, Ideas for a LongBaseline Neutrino Detector,February 1992.

[2] D. Crane and M. Goodman in "Proceedings of Particle and Nuclear Astrophysics and Cosmology in the Next Millenium", June 22-July 14, 1994, Snowmass, Colorado.

[3] K.S.Hirata et al. Phys. Lett. B280, 146 (1992)

[4] D.Casper et al. Phys. Rev. Lett. 66, 2561 (1991) R. Becker-Szendy et al. Phys. Rev. D46, $3720(1992)$

[5] Maury Goodman et al. ANL-HEP-CP-92-124

[6] Ch. Berger et al.,Phys. Lett. B227, 489 (1989), B245, 305 (1990) H. Meyer talk at the 1994 Snowmass Summer Study, July 7, 1994.

[7] Y. Fukuda et al. Phys. Lett. B335, 205 (1994)

[8] G. Rosa et al. CHORUS, a status report. Nucl. Phys. B, Proc. Suppl. : 40 (1995) 85-92

[9] A. Rubbia et al. NOMAD, a status report. Nucl. Phys. B, Proc. Suppl. : 40 (1995) 93-108.

[10] L. Wolfenstein Phys. Rev. D17 (1978) 2369; S.P.Mikheyev and A.Yu. Smirnov, Yad. Fiz. 42 (1985) 1441.

[11] A. Ball et al. SL/Note 92-75(BT)

[12] Benetti P. ICARUS: a neutrino observatory at the Gran Sasso Laboratory. Nucl. Phys. B, Proc. Suppl. : 35 (1994) 280-283

[13] Resvanis, L K. NESTOR: a neutrino particle astrophysics underwater laboratory in the Mediterranean. Proceedings / Neutrino Telescopes, Venice 1993. Ed. by M. Baldo Ceolin

[14] S. Mayoud calculations, unpublished.

[15] P-875 A Long-Baseline Neutrino oscillation Experiment at Fermilab. The MINOS collaboration. Proposal February 1985. Addendum NuMi-L-79 April 1995.

[16] Long Baseline Neutrino Oscillation Experiment E889 Collaboration. BNL No 52459 April 1995.

[17] K. Nishikawa et al. KEK Preprint 93-55.

[18] GEANT 3.21 R. Brun et al. CERN Library

[19] H.C.Fesefeldt. Simulation of hadronic showers, physics and applications. Technical report PITHA 85-02.

[20] A. Fasso et al. FLUKA92. Proceedings of the Workshop on Simulating Accelerator Radiation Environments, Santa Fe, USA, 11-15 January 1993.

[21] Atherton et al. CERN 80-07. Precise measurements of particle production from a $400 \mathrm{GeV} / c$ beam on Beryllium targets.

[22] Barton et al. Fermilab CONF 80-48 EXP

[23] A. Fasso et al. NIM A 332:459, 1993. 

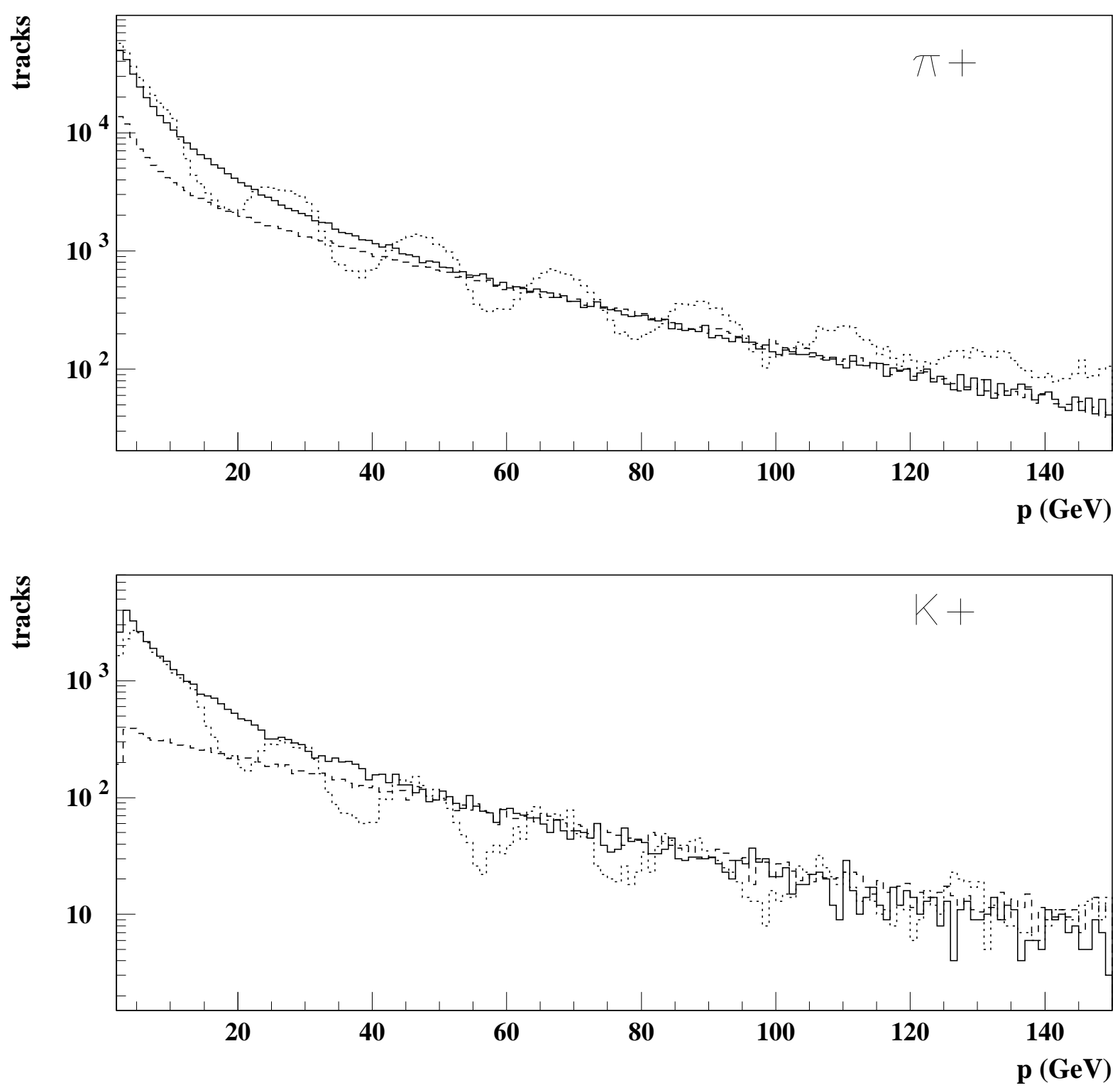

Figure 1: Production spectra of pions and kaons given by the FLUKA(solid), Thermodynamic (dashed) and GHEISHA (dotted) production models (400 GeV/c incident protons) 


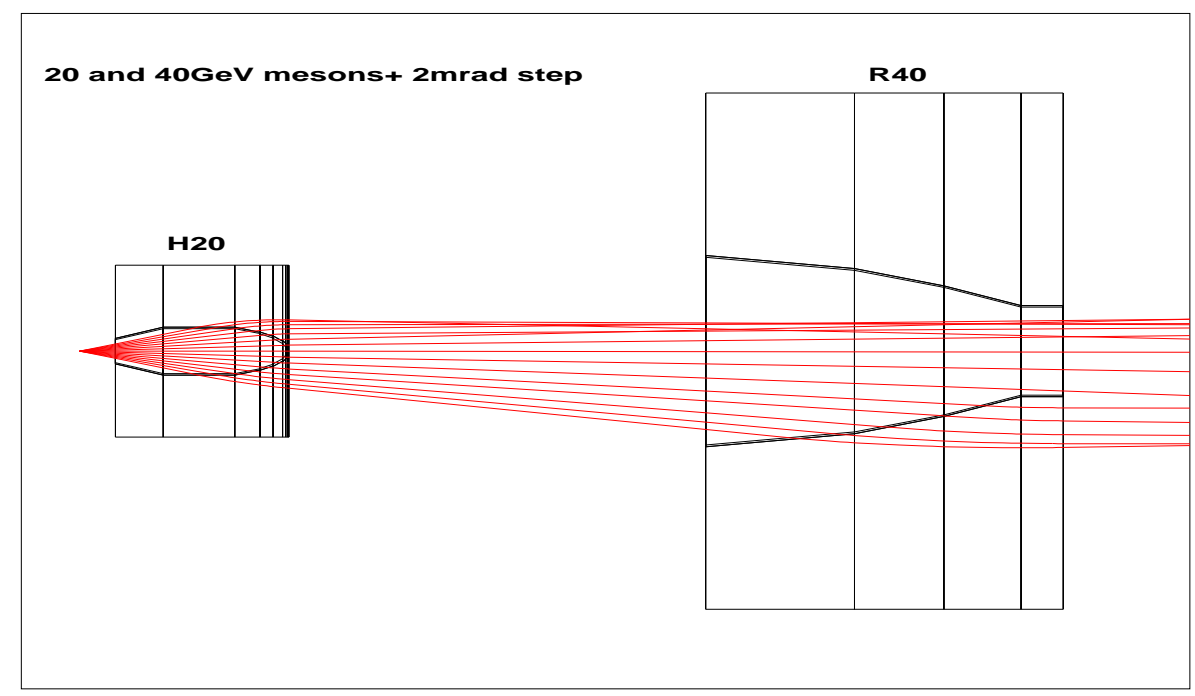

Figure 2: Rays of 20 (upper half) and 40 (lower half) $\mathrm{GeV} / c$ momentum, tracked through the H20R40 horn-reflector focusing system

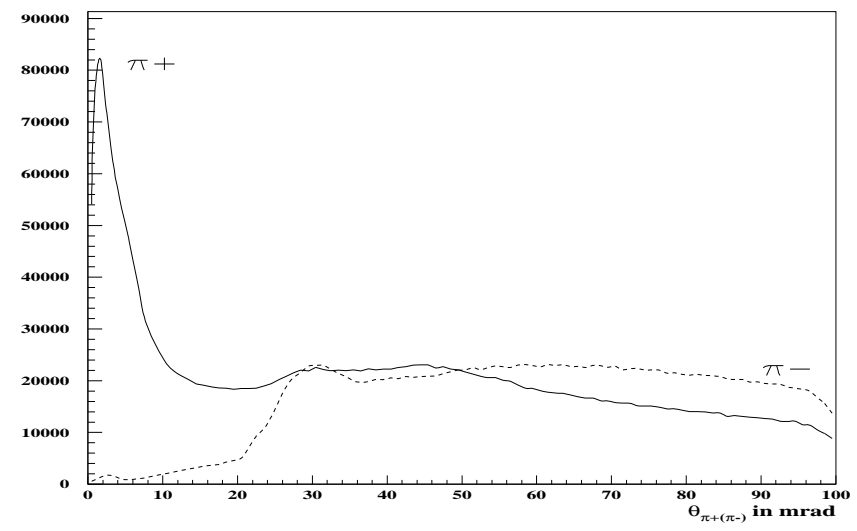

Figure 3: Distribution of the angles of $\pi^{+}$and $\pi^{-}$tracks at the entrance of the decay tunnel showing the focusing/defocusing effect of the H20R40 system for $450 \mathrm{GeV} / c$ incident protons. 

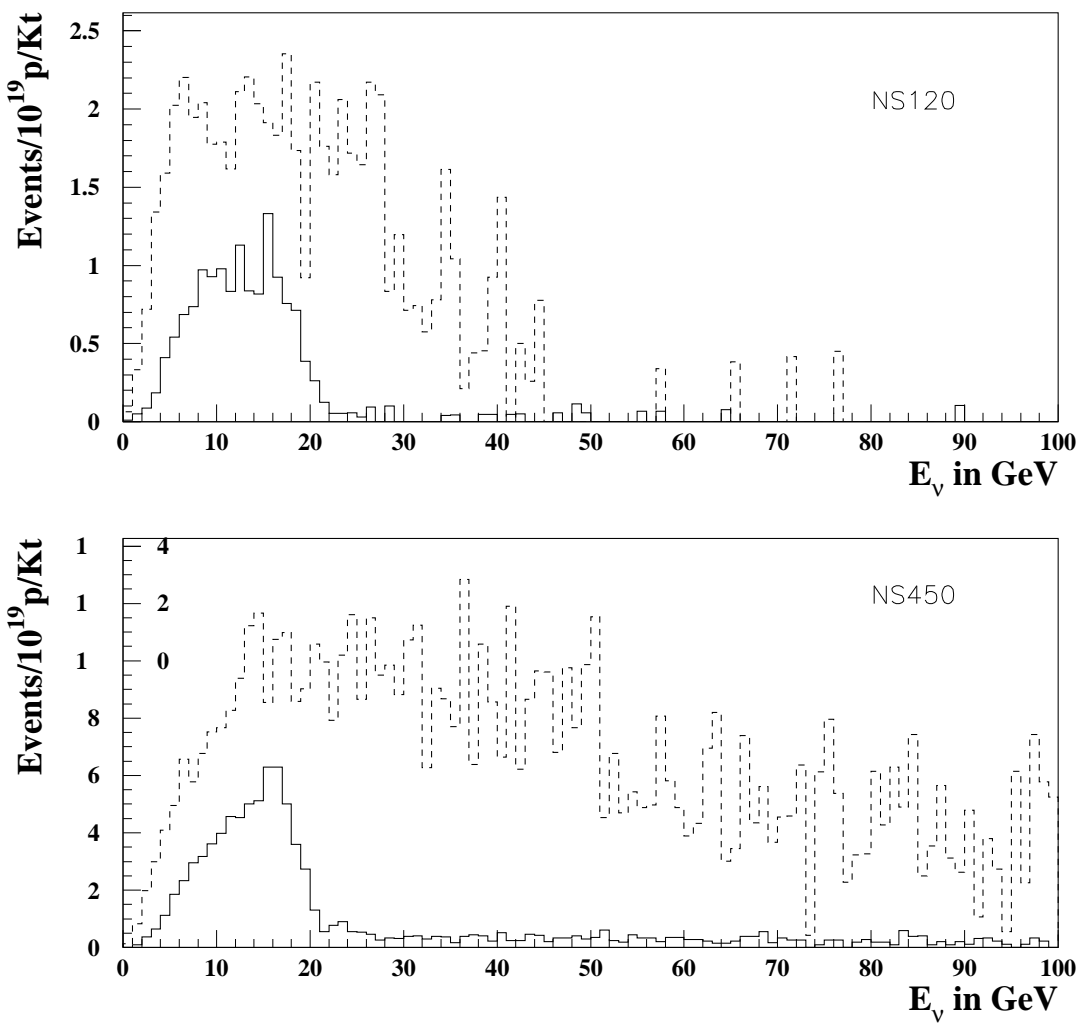

Figure 4: Comparison of the energy distribution of events at NESTOR (NS) for H20R40(solid) and perfect focusing(dashed) with 120 and $450 \mathrm{GeV} / c$ proton beam energies. 

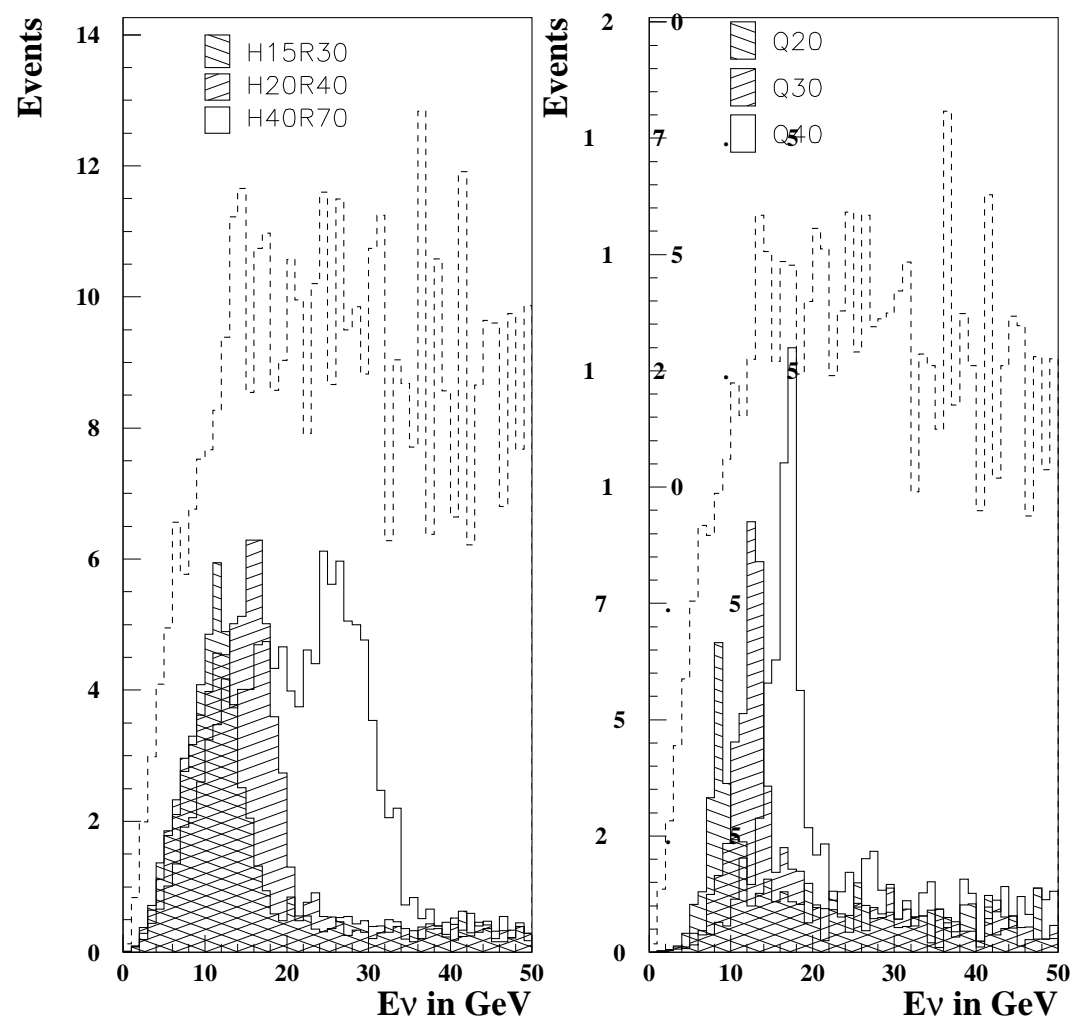

Figure 5: Comparison of the energy distribution of neutrino events at NESTOR for different horn/reflector systems and quadrupole triplets (sum of both neutrino signs) and perfect focusing (dashed lines) (H20R40 focusing and $450 \mathrm{GeV} / c$ beam). 


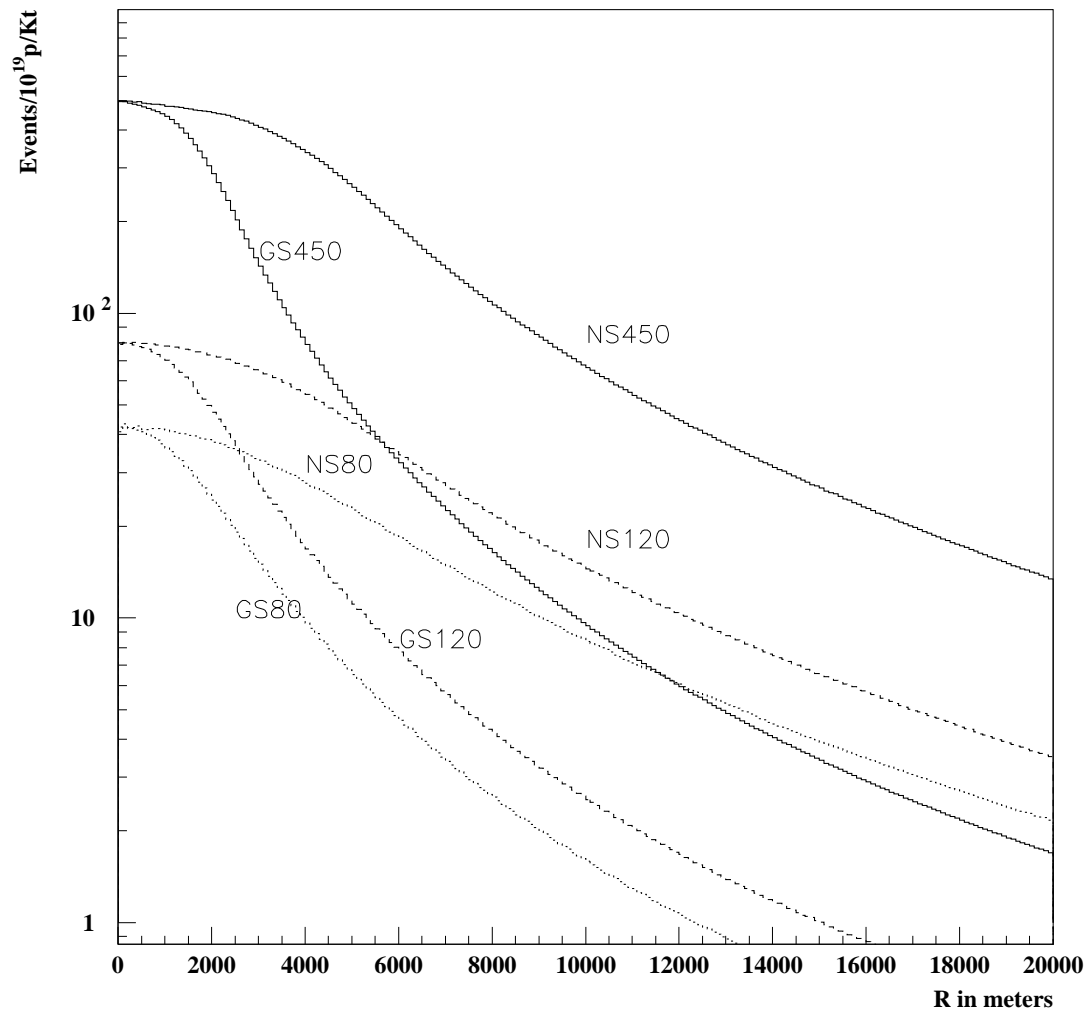

Figure 6: Event radial distributions for incident beam energies of 80,120 and $450 \mathrm{GeV} / c$, at Gran Sasso and NESTOR (NESTOR rates are multiplied by 5.244, see text) (H20R40 focusing and $450 \mathrm{GeV} / c$ beam) 


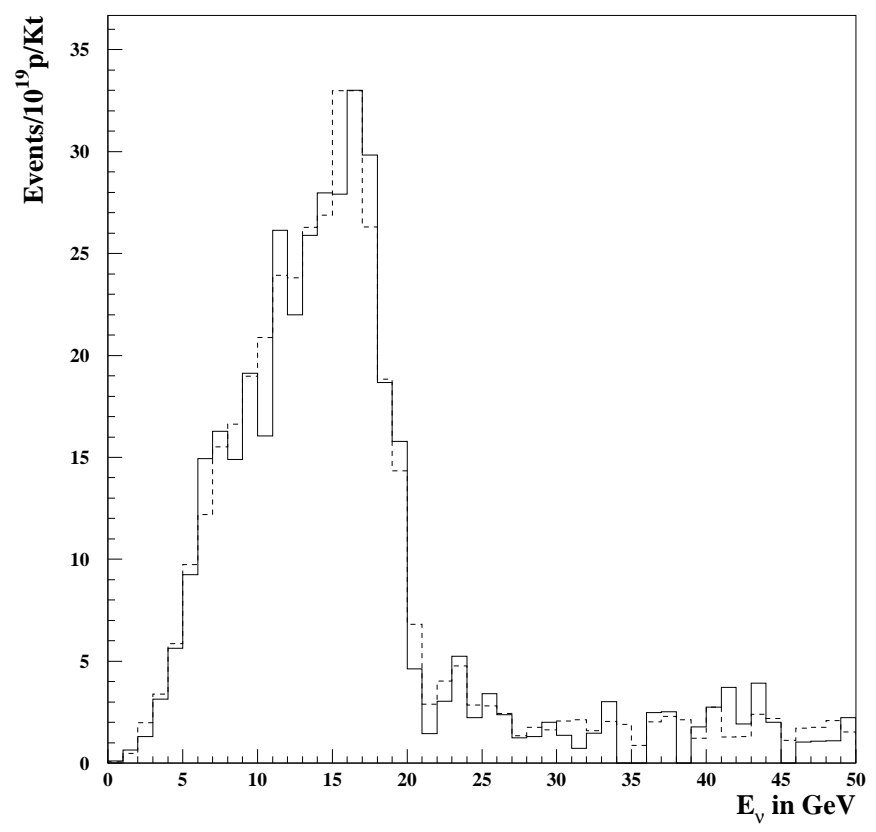

Figure 7: Neutrino event energy distribution for Gran Sasso (solid line) and NESTOR (dashed line) (NESTOR spectrum multiplied by 5.244) (H20R40 focusing and $450 \mathrm{GeV} / \mathrm{c}$ beam) 

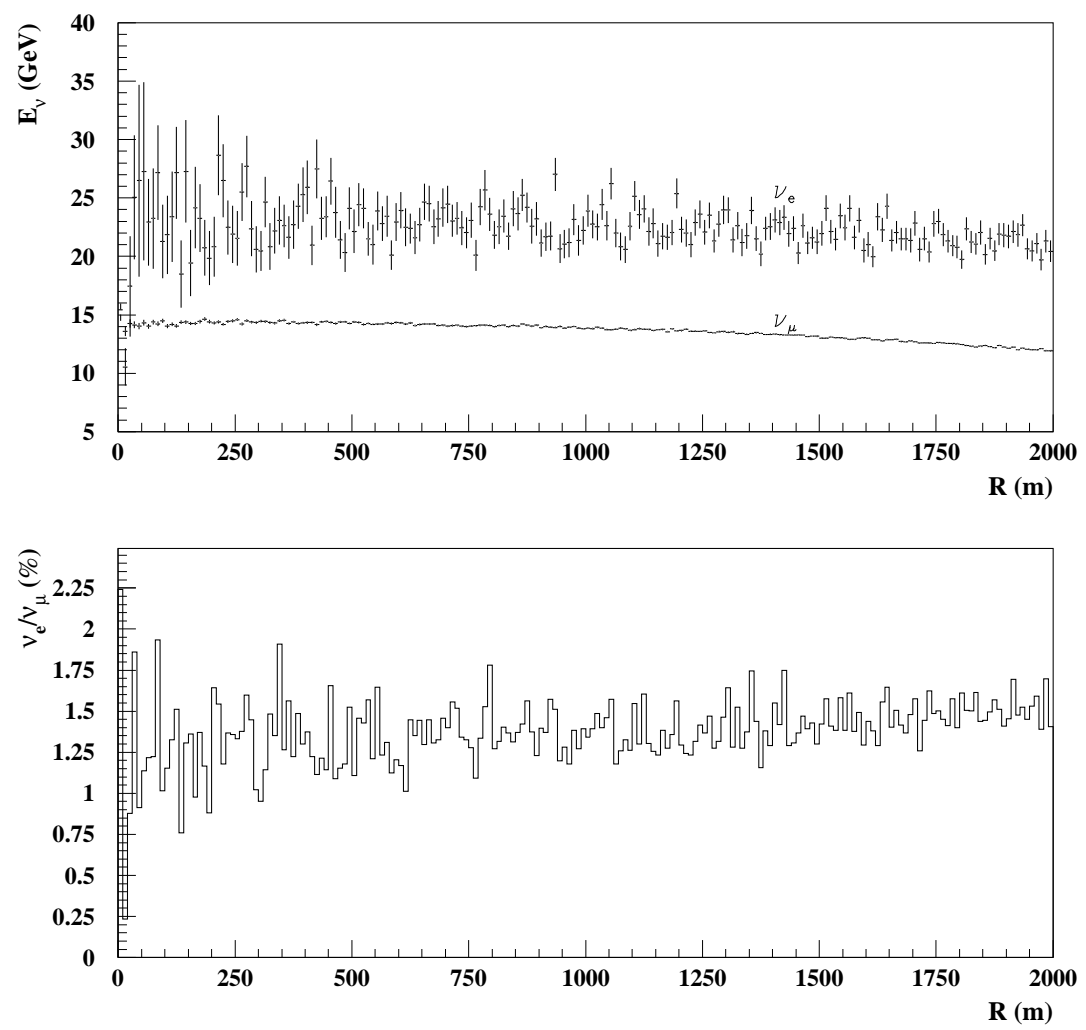

Figure 8: The mean $\nu_{e}$ and $\nu_{\mu}$ energies and the ratio $\nu_{e}$ to $\nu_{\mu}$ as a function of the distance $\mathrm{R}$ from the beam axis at Gran Sasso. (H20R40 focusing and $450 \mathrm{GeV} / c$ beam) 

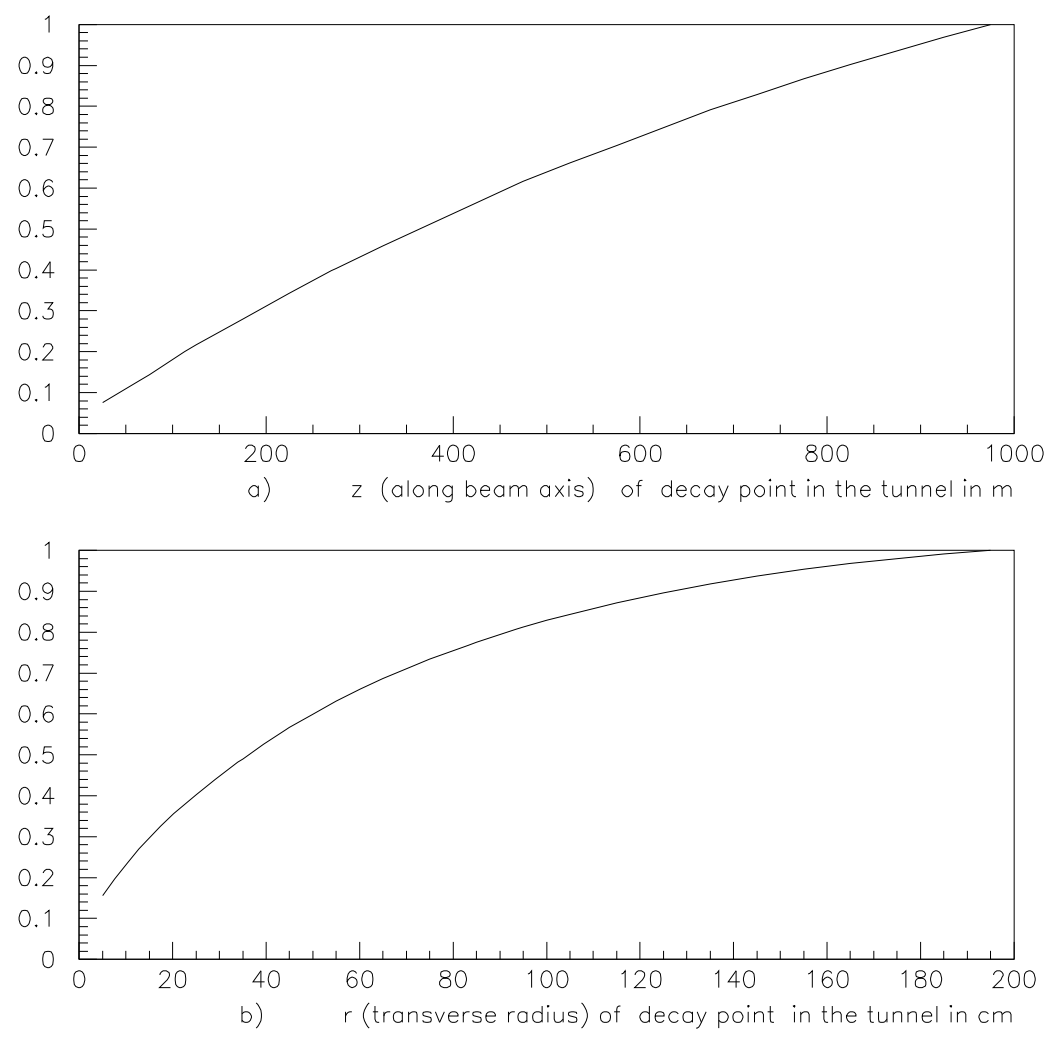

Figure 9: Cumulative A) z distribution and B) radial distance distribution of decay points producing neutrino events inside the fiducial area of NESTOR (H20R40 focusing and $450 \mathrm{GeV} / c$ beam) 

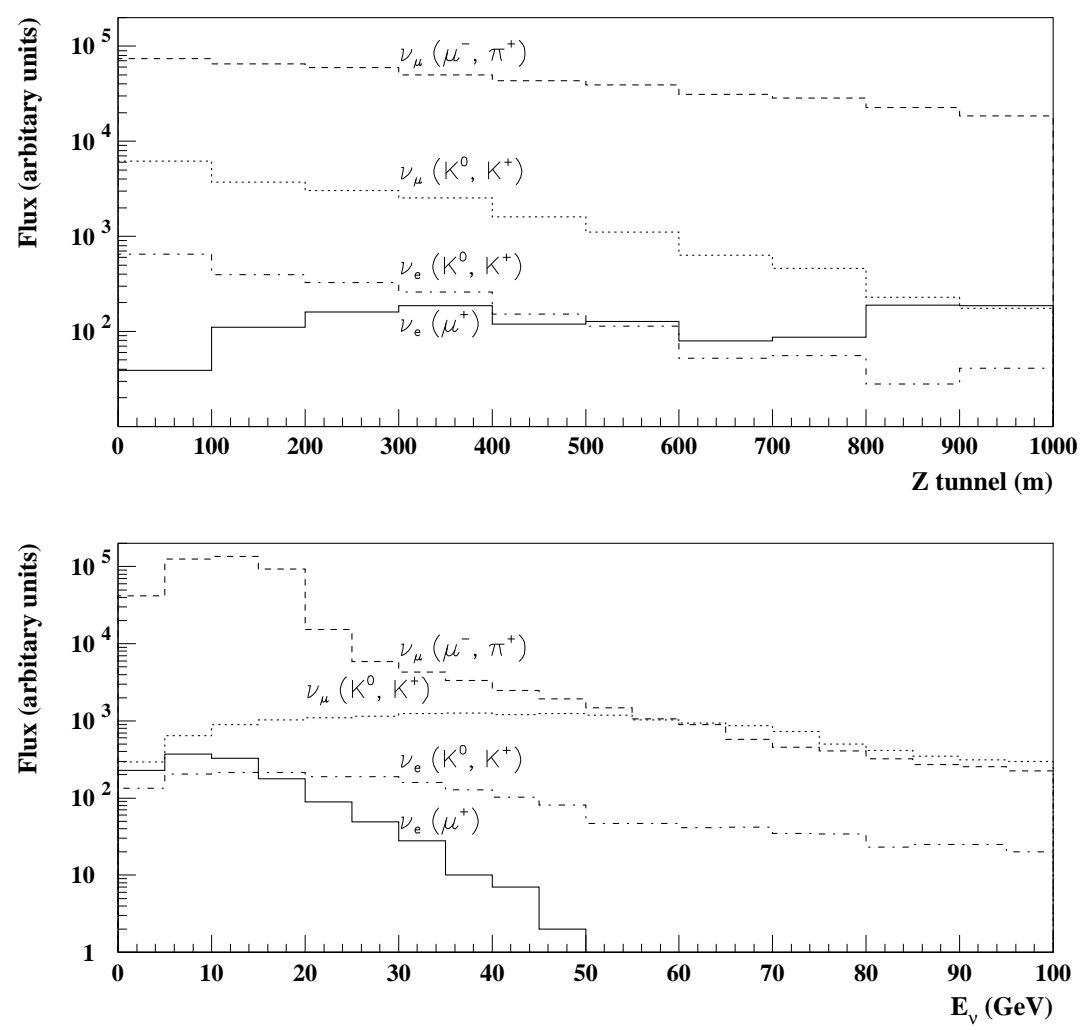

Figure 10: Z distribution along the tunnel of the decay point and neutrino energy distribution of events within the NESTOR acceptance. The identity of the parent particle is denoted in parentheses. (H20R40 focusing and $450 \mathrm{GeV} / c$ beam) 

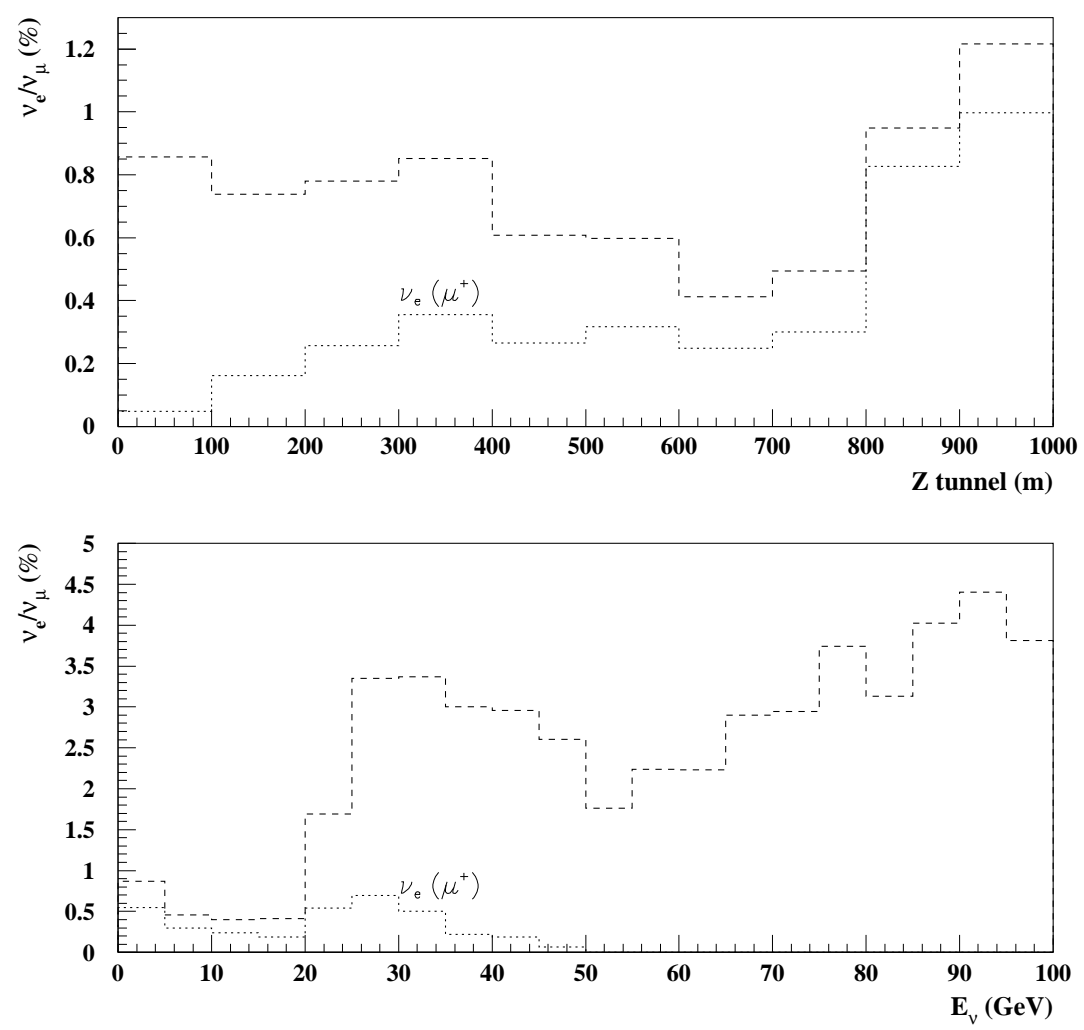

Figure 11: The ratio $\nu_{e}$ to $\nu_{\mu}$ as a function of the $\mathrm{z}$ distribution along the decay tunnel and the neutrino energy, of decays producing events within the NESTOR acceptance. The $\nu_{e}$ coming from $\mu$ are shown separately. (H20R40 focusing and $450 \mathrm{GeV} / c$ beam) 